\title{
INFLUENCE OF DC ELECTRIC FIELD ON THE HYSTERESIS OF LIGHT-INDUCED PACS 61.30.Cz, 61.30.Gd FRÉEDERICKSZ TRANSITION IN A NEMATIC CELL
}

\begin{abstract}
The influence of an external dc electric field on the hysteresis of the light-induced Fréedericksz transition in a nematic liquid crystal cell in the field of a light beam with a finite diameter has been studied. The external electric field orientations perpendicular to the cell surface and along it, in the direction of the incident light polarization, are considered. The dependences of the Fréedericksz transition thresholds for the increasing and decreasing intensities of the incident light on the electric field strength and the transversal light beam size are obtained numerically. The values of transversal light beam size and electric field strength, for which the Fréedericksz transition has a hysteretic character, are found. The hysteresis loop width is shown to increase if the dc electric field perpendicular to the cell surface grows. At the same time, the hysteresis loop width decreases, if the electric field is oriented along the cell surface.

Ke ywords: nematic liquid crystals, light-induced Fréedericksz transition, hysteresis.
\end{abstract}

\section{Introduction}

The rapid development of the liquid crystal (LC) physics within the last decades gave rise to a wide application of LCs in display technologies and hi-tech electron-optical devices [1,2]. A high LC sensitivity to external electric, magnetic, and light fields makes it possible to substantially change the orientational ordering in the mesophase and, hence, the electro- and magneto-optical properties of specimens [3-9]. The optical nonlinearity of LCs opened prospects for their wide application in photonics [10].

In particular, a threshold orientational instability of the director can arise in an LC cell under the light field action, the so-called light-induced Fréedericksz transition (LIFT) [11]. An important characteristic of the LIFT is the threshold value $I_{\mathrm{th}}$ for the incident light intensity, above which the LC director smoothly transits from the homogeneous state into an inhomogeneous one or vice versa, if the light intensity decreases below it. The theory of LIFT in nematic liquid crystals (NLCs) has been developed by a number of authors [12-15]. However, it was found in some works $[13,16,17]$ that, under certain conditions, the threshold value $I_{\mathrm{th}}$ of direc-

(C) M.F. LEDNEY, O.S. TARNAVSKYY,

V.V. KHIMICH, 2016

ISSN 2071-0194. Ukr. J. Phys. 2016. Vol. 61, No. 2 tor orientational instability obtained, when the incident light intensity grows, can differ from the corresponding value $I_{\text {th }}^{\prime}$ obtained, when the light intensity decreases. The director transition from the homogeneous state into an inhomogeneous one and vice versa, when the light intensity attains the threshold values, occurs in a jump-like manner, i.e. the LIFT is accompanied by a hysteresis. For the first time, the hysteresis of the LIFT was experimentally observed in an NLC cell in the field of a linearly polarized light beam and in the presence of an additional dc magnetic field [18]. The LIFT hysteresis in the field of a linearly polarized light, which could be controlled by a quasi-static electric field, was studied experimentally in both homeotropic [19-21] and planar-oriented NLCs [22-24]. The LIFT hysteresis under crossed electric and magnetic fields was considered in work [25]. Note that additional external dc fields do not create a hysteresis of the LIFT, but only expand the region of its existence, which allows the hysteresis to be registered experimentally. In work [26], the optical multistability of NLCs at the LIFT was predicted for the first time; later, it was studied both experimentally and theoretically [2729]. The LIFT in the presence of a hysteresis in NLCs doped with dendrimers was studied experimentally in work [30]. 
The influence of the finite transverse size of a light beam on the LIFT threshold - however, in the absence of a hysteresis - was theoretically studied in the NLC cell with both infinite [13,15] and finite [31] energies of the director anchoring at the surface. The finite transverse size of a light beam was taken into account, while experimentally studying the LIFT hysteresis [18, 20-23]. The influence of the shape and the finite transverse light beam size on parameters of the LIFT hysteresis in an NLC cell with an infinitely rigid surface anchoring, but in the absence of external dc fields, was studied theoretically in works $[32,33]$. The influence of a finite energy of anchoring of NLCs with the cell surface on parameters of the LIFT hysteresis was studied theoretically in the fields of both an infinitely wide light beam $[16,34]$ and a light beam with a finite width.

In this work, a possibility to control the hysteresis of the LIFT in the field of a light beam with a finite transverse size by applying a dc electric field to the NLC cell is considered theoretically.

\section{Equations for the Director and Their Solution}

Let us consider a plane-parallel NLC cell, which is confined between the planes $z=0$ and $z=L$, with the initially homogeneous and homeotropic director orientation along the axis $O z$. The NLC anchoring with the cell surface is considered to be infinitely rigid. The cell is embedded into an external uniform dc electric field with the strength vector $\mathbf{E}_{0}=\left(E_{0}, 0,0\right)$ directed along the axis $O x$. A monochromatic light wave linearly polarized along the axis $O x$ falls on the cell along the axis $O z$. The electric field of the wave has the form

$\mathbf{E}=\frac{1}{2}\left[\mathbf{E}(\mathbf{r}) e^{-i \omega t}+\mathbf{E}^{*}(\mathbf{r}) e^{i \omega t}\right]$.

For definiteness, let the incident light beam be confined along the axis $O y$, and let the intensity distribution in its transverse cross-section be described by the function

$I(y)=I_{0} \Theta(a-|y|)$,

where $\Theta(x)=1$ at $x \geqslant 0$ and 0 at $x<0$, and $2 a$ is the light beam width.

The free energy of an NLC cell can be written in the form

$F=F_{\mathrm{el}}+F_{E}+F_{E}^{0}$, where

$F_{\text {el }}=\frac{1}{2} \int_{V}\left\{K_{1}(\operatorname{div} \mathbf{n})^{2}+K_{2}(\mathbf{n} \cdot \operatorname{rot} \mathbf{n})^{2}+\right.$
$\left.+K_{3}[\mathbf{n} \times \operatorname{rot} \mathbf{n}]^{2}\right\} d V$,

is the Frank elastic energy and

$F_{E}=-\frac{1}{16 \pi} \int_{V} \varepsilon_{i j} E_{i} E_{j}^{*} d V$,
$F_{E}^{0}=-\frac{\varepsilon_{a}^{0}}{8 \pi} \int_{V}\left(\mathbf{n E}_{0}\right)^{2} d V$,

are anisotropic contributions to the NLC free energy by the electric field of a light wave [15] and the dc electric field [1], respectively; $\mathbf{n}$ is the director; $K_{1}, K_{2}$, and $K_{3}$ are the NLC elastic constants; $\varepsilon_{i j}=\varepsilon_{\perp} \delta_{i j}+\varepsilon_{a} n_{i} n_{j}$ and $\varepsilon_{a}=\varepsilon_{\|}-\varepsilon_{\perp}>0$ are the tensor and the anisotropy, respectively, of the NLC dielectric permittivity at the frequency of an incident light; $\varepsilon_{a}^{0}=\varepsilon_{\|}^{0}-\varepsilon_{\perp}^{0}>0$ is the anisotropy of the static dielectric permittivity; and $\varepsilon_{\|}, \varepsilon_{\perp}, \varepsilon_{\|}^{0}$, and $\varepsilon_{\perp}^{0}$ are the principal values of corresponding dielectric tensors for a homogeneous nematic in the parallel and perpendicular directions with respect to the director.

Only planar deformations of the NLC director near the orientational instability threshold [15] will be considered. In this case, owing to the uniformity along the axis $O x$, the director in the NLC bulk in the Cartesian coordinates has the form

$\mathbf{n}=\mathbf{e}_{x} \cdot \sin \varphi(y, z)+\mathbf{e}_{z} \cdot \cos \varphi(y, z)$,

where $\varphi$ is the angle of the director deviation from its initial homogeneous direction along the axis $O z$. Minimizing the free energy (2) of the NLC cell by varying the angle $\varphi$, we obtain the following stationary equation:

$\left(1-k \sin ^{2} \varphi\right) \frac{\partial^{2} \varphi}{\partial z^{2}}+m \frac{\partial^{2} \varphi}{\partial y^{2}}-k \sin \varphi \cos \varphi\left(\frac{\partial \varphi}{\partial z}\right)^{2}+$

$+\frac{\pi^{2}}{L^{2}} \frac{I_{0}}{I_{\mathrm{Fr}}} \frac{\varepsilon_{\|}^{3 / 2} \sin \varphi \cos \varphi}{\left(\varepsilon_{\perp}+\varepsilon_{a} \cos ^{2} \varphi\right)^{3 / 2}} \times$

$\times \Theta(a-|y|)+\frac{\pi^{2}}{L^{2}} \frac{E_{0}^{2}}{E_{0 \mathrm{Fr}}^{2}} \sin \varphi \cos \varphi=0$,

where

$I_{\mathrm{Fr}}=\frac{8 \pi^{3} \varepsilon_{\|} K_{3}}{\varepsilon_{a} \varepsilon_{\perp} L^{2}}, E_{0 \mathrm{Fr}}=\sqrt{\frac{4 \pi^{3} K_{3}}{\varepsilon_{a}^{0} L^{2}}}$

ISSN 2071-0194. Ukr. J. Phys. 2016. Vol. 61, No. 2 
are the Fréedericksz transition thresholds in the field of a uniform, infinitely wide light beam [13] and in the dc electric field $[25,36]$, respectively, provided an infinitely rigid NLC anchoring with the cell surface; $k=\left(K_{3}-K_{1}\right) / K_{3}$; and $m=K_{2} / K_{3}$. Equation (4) involves the solutions of Maxwell's equations for the electric field of an incident light wave and the dc electric field in the nematic volume. In particular, in the geometric optics approximation, the electric field of a light beam has the form [32]

$$
\begin{aligned}
& E_{x}(y, z)=I^{1 / 2}(y)\left(\frac{\varepsilon_{z z}}{\varepsilon_{\|}}\right)^{1 / 4} \times \\
& \times \exp \left[i \frac{\omega}{c} \sqrt{\varepsilon_{\perp} \varepsilon_{\|}} \int_{0}^{z} \varepsilon_{z z}^{-1 / 2} d z^{\prime}\right], \\
& E_{z}(y, z)=-\frac{\varepsilon_{z x}}{\varepsilon_{z z}} E_{x}(y, z),
\end{aligned}
$$

where $\varepsilon_{z z}=\varepsilon_{\perp}+\varepsilon_{a} \cos ^{2} \varphi$, and $\varepsilon_{z x}=\varepsilon_{a} \cos \varphi \sin \varphi$. From the electrostatics equations and taking the continuity of the tangential components of the dc electric field across the cell surface into consideretion, it follows that $E_{0 x}=E_{0}$.

Equation (4) together with the boundary conditions $\varphi(z=0, L)=0$ corresponding to the infinitely rigid anchoring of an NLC at the cell surface are considered in a vicinity of the director orientational instability threshold. Therefore, deformations of the director field are assumed to be small. The director deviation angle $\varphi$ and the incident light intensity $I_{0}$ are expanded in series in the small parameter [32], namely, the maximum of the director deviation angle $\varphi_{m}=\varphi(y=0, z=L / 2)$. This maximum is reached at the cell midpoint and the center of the light beam. Since the direction of a director deviation is governed by director fluctuations only and does not depend on the incident light intensity $I_{0}$, the expansion for the intensity $I_{0}$ has the form

$$
\frac{I_{0}}{I_{\mathrm{Fr}}}=\rho+\sigma \varphi_{m}^{2}+\tau \varphi_{m}^{4}+o\left(\varphi_{m}^{4}\right),
$$

where the unknown expansion coefficients $\rho, \sigma$, and $\tau$ are determined by the light beam shape and depend on the dc electric field strength.

Since the signs of the angles $\varphi$ and $\varphi_{m}$ are identical, the solution of Eq. (4) is sought in the form of a series expansion in odd powers of $\varphi_{m}$ :

$\varphi(y, z)=A(y, z) \varphi_{m}+B(y, z) \varphi_{m}^{3}+$

ISSN 2071-0194. Ukr. J. Phys. 2016. Vol. 61, No. 2
$+C(y, z) \varphi_{m}^{5}+o\left(\varphi_{m}^{5}\right)$.

Here, the unknown expansion coefficients $A(y, z)$, $B(y, z)$, and $C(y, z)$ satisfy the condition of infinitely rigid NLC anchoring with the cell surface,

$A(y ; z=0, L)=B(y ; z=0, L)=$

$=C(y ; z=0, L)=0$

and the conditions following from the definition of the director deviation angle maximum,

$$
\begin{aligned}
& A(y=0, z=L / 2)=1, \\
& B(y=0, z=L / 2)=C(y=0, z=L / 2)=0 .
\end{aligned}
$$

In view of expansions (6) and (7), let us rewrite Eq. (4) to an accuracy order of the $\varphi_{m}^{5}$-terms inclusive. By equating the coefficients in front of the powers of $\varphi_{m}$ to zero, we obtain the following system of differential equations for the functions $A(y, z)$, $B(y, z)$, and $C(y, z)$ :

$$
\begin{aligned}
& A_{z z}^{\prime \prime}+m A_{y y}^{\prime \prime}+\frac{\pi^{2}}{L^{2}}\left(\rho \Theta(a-|y|)+\rho_{0}\right) A=0 \\
& B_{z z}^{\prime \prime}+m B_{y y}^{\prime \prime}+\frac{\pi^{2}}{L^{2}}\left(\rho \Theta(a-|y|)+\rho_{0}\right) B= \\
& =k A^{2} A_{z z}^{\prime \prime}+k A_{z}^{\prime 2} A-\frac{\pi^{2}}{L^{2}}\left[\left(\rho \alpha A^{3}+\sigma A\right) \times\right. \\
& \left.\times \Theta(a-|y|)-\frac{2}{3} \rho_{0} A^{3}\right] \\
& C_{z z}^{\prime \prime}+m C_{y y}^{\prime \prime}+\frac{\pi^{2}}{L^{2}}\left(\rho \Theta(a-|y|)+\rho_{0}\right) C= \\
& k A^{2} B_{z z}^{\prime \prime}+2 k A B A_{z z}^{\prime \prime}-\frac{k}{3} A^{4} A_{z z}^{\prime \prime}+ \\
& +k A_{z}^{\prime 2} B-\frac{2}{3} k A_{z}^{\prime 2} A^{3}+2 k A_{z}^{\prime} B_{z}^{\prime} A- \\
& -\frac{\pi^{2}}{L^{2}}\left[\left(3 \rho \alpha A^{2} B+\rho \beta A^{5}+\sigma B+\sigma \alpha A^{3}+\right.\right. \\
& \left.+\tau A) \Theta(a-|y|)-2 \rho_{0} A^{2} B+\frac{2}{15} \rho_{0} A^{5}\right] .
\end{aligned}
$$

Here, $u=\frac{\varepsilon_{a}}{\varepsilon_{\|}}, \alpha=\frac{3}{2} u-\frac{2}{3}, \beta=\frac{15}{8} u^{2}-\frac{3}{2} u+\frac{2}{15}, \rho_{0}=$ $=\frac{E_{0}^{2}}{E_{0 \mathrm{Fr}}^{2}}$, and the primed $A, B$, and $C$ functions mean their differentiation with respect to the corresponding coordinates.

From the condition that the solution of system (10)-(12) should satisfy Eqs. (8) and (9), we determine the coefficients $\rho, \sigma$, and $\tau$ in expansion (6). 
They depend on the NLC cell parameters, the shape and the transverse size of an incident light beam, and the external electric field strength. From expansion (6), to an accuracy order of the $\varphi_{m}^{4}$-terms, we obtain

$\varphi_{m}^{2}=\frac{1}{2 \tau}\left[-\sigma \pm \sqrt{\sigma^{2}+4 \tau\left(\frac{I_{0}}{I_{\mathrm{Fr}}}-\rho\right)}\right]$.

From whence, it follows that, if $\sigma>0$, the LIFT occurs without the hysteresis. When the incident light intensity $I_{0}$ achieves the orientational instability threshold $I_{\mathrm{th}}=\rho I_{\mathrm{Fr}}$, the system smoothly transits from the homogeneous state with $\varphi_{m}=0$ into an inhomogeneous one with $\varphi_{m} \neq 0$, and vice versa (see works $[16,32])$.

Let $\sigma<0$. Then the LIFT is accompanied by the hysteresis. In particular, if the incident light intensity $I_{0}$ increases from zero and achieves the value of $I_{\mathrm{th}}$, the system, according to Eq. (13) [16,32], transits in a jump-like manner from the homogeneous state with $\varphi_{m}=0$ into an inhomogeneous one with $\varphi_{m}==\sqrt{-\frac{\sigma}{\tau}}$. On the other hand, if the intensity $I_{0}$ decreases from values higher than $I_{\mathrm{th}}$, the system returns into the initial homogeneous state from the state with $\varphi_{m}=\sqrt{-\frac{\sigma}{2 \tau}}$ at the light intensity $I_{\mathrm{th}}^{\prime}=I_{\mathrm{th}}-I_{\mathrm{Fr}} \sigma^{2} /(4 \tau)<I_{\mathrm{th}}{ }^{2 \tau}$ (see Eq. (13) and works $[16,32])$. The width of the hysteresis loop amounts to $\Delta I_{\mathrm{th}}=I_{\mathrm{Fr}} \sigma^{2} /(4 \tau)>0$.

Hence, the parameters $\rho, \sigma$, and $\tau$ determine the threshold character of the LIFT (the presence or the absence of hysteresis) and the hysteresis parameters, namely, the threshold values, the hysteresis loop width, and the jump magnitude for the director deviation angle maximum, when the incident light intensity achieves the threshold values.

For a non-trivial solution $A(y, z)$ of Eq. (10) to exist, which would be finite at $y \rightarrow \pm \infty$, continuous at $y= \pm a$, and satisfying conditions (8) and (9), the following equation for the determination of the parameter $\rho$ is obtained:

$\xi \tan \xi=q a$,

where $q=\frac{\pi}{L \sqrt{m}}$, and $\xi=q a \sqrt{\rho+\rho_{0}-1}$.

From Eq. (11), in which $A(y, z)$ is the solution of Eq. (10), and taking conditions (8) and (9) into account, we find the parameter $\sigma$ :

$\sigma=-\frac{3}{16}\left(2 k+3 \alpha \rho-2 \rho_{0}\right)-$ $-\left(16\left(k-\rho_{0}\right) s^{2} \cos ^{3} \xi-\left(2 k+3 \alpha \rho-2 \rho_{0}\right) \times\right.$

$\times(\cos 3 \xi-3 s \sin 3 \xi)) /(64(\xi \sin \xi+s \sin \xi+s \xi \cos \xi))$,

where $s=\sqrt{\frac{\rho+\rho_{0}-1}{1-\rho_{0}}}$.

An explicit form for the parameter $\tau$ is too cumbersome, and it is not presented here.

\section{Hysteresis of Light-Induced Fréedericksz Transition}

\subsection{Electric field} parallel to the cell surface

Let us consider the case where the external dc electric field is oriented along the cell surface and, simultaneously, in the direction of the incident light polarization vector. The dependences of the LIFT threshold values for the increasing, $I_{\mathrm{th}}=\rho I_{\mathrm{Fr}}$, and decreasing, $I_{\mathrm{th}}^{\prime}=\left[\rho-\sigma^{2} /(4 \tau)\right] I_{\mathrm{Fr}}$, incident light intensities on the light beam half-width $a$ calculated for various dc electric field strengths $E_{0}$ 's are depicted in Fig. 1, a. For calculations, we used the NLC parameter values close to typical ones: $k=0.8, m=0.3$, $\varepsilon_{\|}=3.06$, and $\varepsilon_{\perp}=2.37$ [1], As the beam width increases, the magnitudes of both thresholds, $I_{\text {th }}$ and $I_{\mathrm{th}}^{\prime}$, monotonically decrease at all electric field strengths $E_{0}$. But the difference $\Delta I_{\text {th }}=I_{\text {th }}-I_{\text {th }}^{\prime}$ between the threshold values, i.e. the hysteresis loop width, depends non-monotonically on the transverse size $2 a$ of a light beam (see Fig. 1, b), as the intensity increases or decreases. When $a$ grows, the value of $\Delta I_{\mathrm{th}}$ first increases from 0 for a narrow beam to a certain maximum. Then it decreases to a certain nonzero value for an infinitely wide $(a \rightarrow \infty)$ beam.

Hence, the hysteresis loop as a function of the light beam width has a maximum. This maximum is reached for light beams with the transverse size of an order of the thickness of a few cells. For the NLC parameters indicated above, the corresponding half-width of the incident light beam amounts to $a \approx 3 L$. With the growth of the external electric field strength $E_{0}$, the position of the hysteresis loop width maximum weakly shifts toward wider light beams.

The dc electric field evidently favors the reorientation of the NLC director. Therefore, irrespective of the transverse light beam size, the magnitudes of both thresholds, $I_{\mathrm{th}}$ and $I_{\mathrm{th}}^{\prime}$, decrease, when the electric field strength $E_{0}$ grows, and vanish, when the strength achieves the Fréedericksz transition threshold, $E_{0}=E_{0 \mathrm{Fr}}$. The hysteresis loop of the LIFT

ISSN 2071-0194. Ukr. J. Phys. 2016. Vol. 61, No. 2 
gets narrower monotonically with the growth of the electric field strength $E_{0}$. At a certain critical value $E_{0}^{*} \leqslant E_{0 \mathrm{Fr}}$, which depends on the NLC parameters and the light beam width, the hysteresis disappears (Fig. 2). If the dc electric field strength falls within the interval $E_{0}^{*} \leqslant E_{0} \leqslant E_{0 \mathrm{Fr}}$, the LIFT occurs without the hysteresis.

As one can see from Fig. 1, $b$, the LIFT is accompanied by the hysteresis if the half-width $a$ of the incident light beam exceeds the critical value $a_{\mathrm{th}}$ (this is the value of $a$, which corresponds to $\sigma=0$ ), i.e. $a>a_{\mathrm{th}}$. In the light beam with $a<a_{\mathrm{th}}$, the LIFT occurs without the hysteresis. The dependence of the critical value of the light beam half-width $a_{\text {th }}$ on the electric field strength $E_{0}$ is depicted in Fig. 3, a. One can see that there exists another critical value for the electric field strength,

$E_{0}^{* *}=E_{0 \mathrm{Fr}} \sqrt{\frac{4 \varepsilon_{\|}}{9 \varepsilon_{a}}(k-1)+1}$,

which corresponds to $\sigma=0$ as $a \rightarrow \infty$. If $E_{0}<E_{0}^{* *}$, the LIFT is accompanied by the hysteresis in light beams with the half-width $a>a_{\mathrm{th}}$. If the electric field strength $E_{0}>E_{0}^{* *}$, the LIFT hysteresis is absent in light beams with arbitrary transverse sizes. When the parameter $k$ grows, the region of the LIFT hysteresis existence expands with respect to both the electric field strength $E_{0}$ and the transverse light beam size $2 a$.

The finite width of the incident light beam substantially affects not only the magnitudes of LIFT hysteresis parameters, but also the conditions of hysteresis existence, similarly to the case of the dc electric field absence, $E_{0}=0[32,33]$. For a fixed ratio $a / L$, the LIFT is accompanied by the hysteresis $(\sigma<0)$ in the intervals of parameters $k_{\mathrm{th}}<k<1$ and $0<m<m_{\mathrm{th}}$. If $k<k_{\mathrm{th}}$ and $m>m_{\mathrm{th}}$, the LIFT occurs without the hysteresis $(\sigma>0)$. The dependences of the critical parameter values $k_{\mathrm{th}}$ and $m_{\mathrm{th}}$ (these are $k$ - and $m$-values, which correspond to $\sigma=0$ ) on the transverse light beam size are qualitatively similar to those obtained in the absence of an external electric field $\mathbf{E}_{0}[32,33]$.

The intervals of $k$ - and $m$-values, where the LIFT hysteresis exists, grow with the increase of the transverse light beam size irrespective of the applied electric field strength $E_{0}$. The dependences of the critical value of parameter $m_{\mathrm{th}}$ on the dc electric field
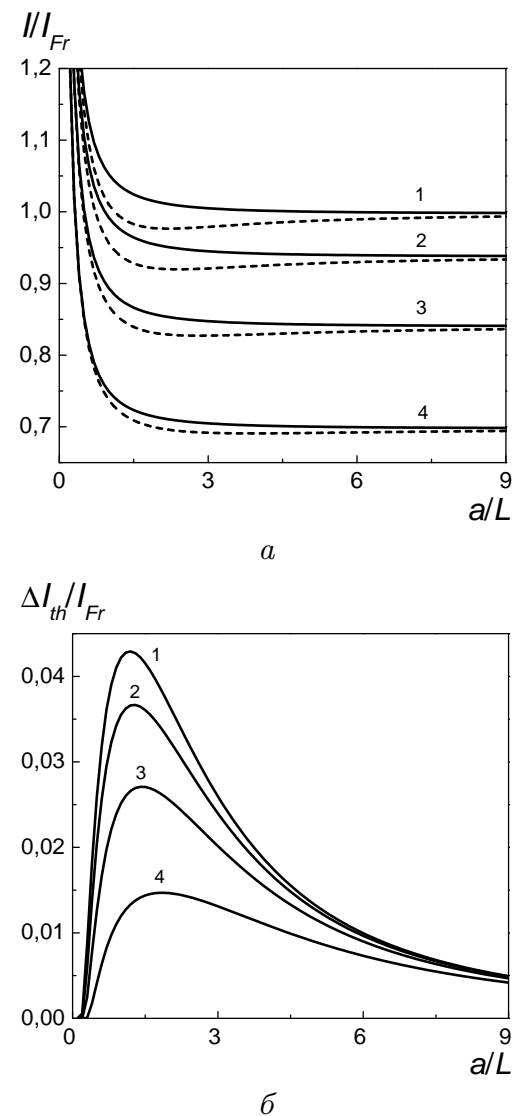

Fig. 1. LIFT threshold $I_{\text {th }}$ at the increasing (solid curves) and decreasing (dashed curves) electric field strengths $(a)$ and the hysteresis loop width $\Delta I_{\text {th }}$ versus the transverse light beam size for various $E_{0} / E_{0 \mathrm{Fr}}=0.05$ (1), 0.25 (2), 0.4 (3), and $0.55(4)(b)$

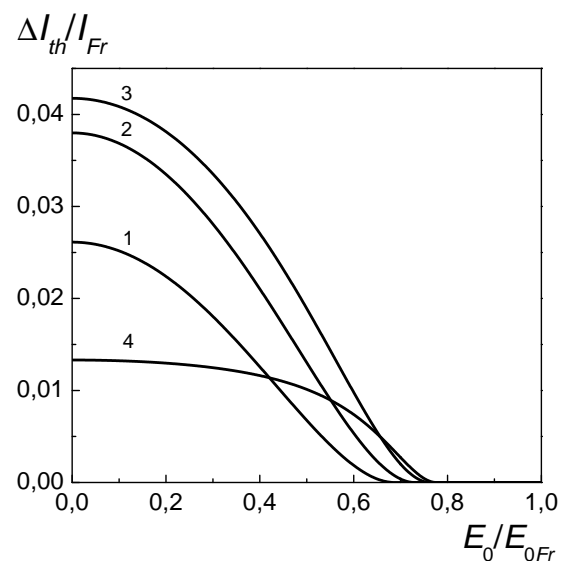

Fig. 2. Dependences of the LIFT hysteresis loop width $\Delta I_{\text {th }}$ on the electric field strength $E_{0}$ directed along the cell surface. $a / L=0.5$ (1), 0.75 (2), 1 (3), and 5 (4) 

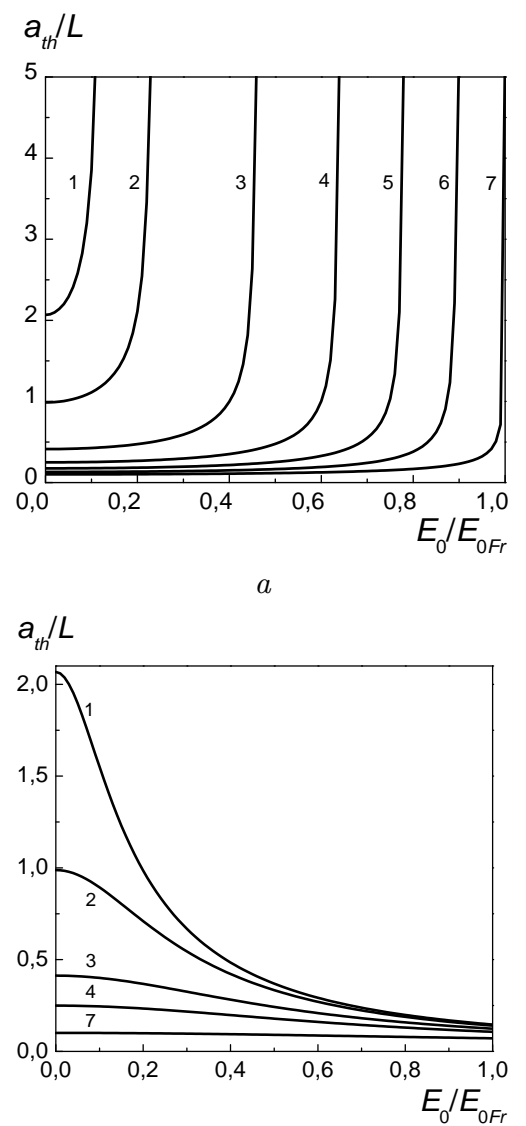

$b$

Fig. 3. The critical light beam half-width $a_{\mathrm{th}}$ versus the strength $E_{0}$ of electric field directed along (a) and perpendicularly (b) to the cell surface. $m=0.3 ; k=0.5$ (1), 0.52 (2), 0.6 (3), $0.7(4), 0.8(5), 0.9(6)$, and 1 (7)

strength $E_{0}$ are depicted in Fig. 4, $a$ for various transverse light beam sizes. The region of the allowed values of parameter $m$, at which the LIFT is accompanied by the hysteresis $\left(0<m<m_{\text {th }}\right)$, gets narrower, when the electric field strength $E_{0}$ increases, and, at $E_{0} \geq E_{0}^{*}$, the hysteresis disappears. If the electric field strength $E_{0}$ increases, the interval of $k$-values, where the LIFT hysteresis exists $\left(k_{\mathrm{th}}<k<1\right)$, also gets narrower irrespective of the transverse light beam size.

Hence, the growth of the dc electric field strength $E_{0}$ in the case where the field is oriented along the cell surface and in the direction of the incident light polarization vector gives rise to a reduction of the threshold magnitudes $I_{\text {th }}$ and $I_{\mathrm{th}}^{\prime}$, and the LIFT hysteresis loop width $\Delta I_{\mathrm{th}}$. At a certain strength value, the hys-
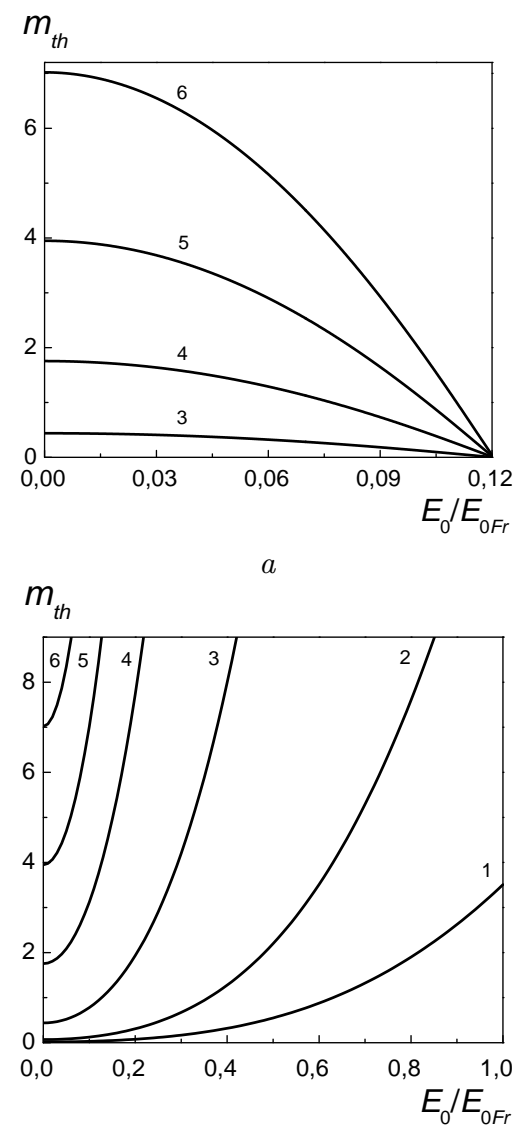

$b$

Fig. 4. The critical value for the parameter $m$ versus the strength $E_{0}$ of the dc electric field directed along (a) and perpendicularly (b) to the cell surface. $k=0.5 ; a / L=0.5$ (1), 1 (2), 2.5 (3), 5 (4), $7.5(5)$, and $10(6)$

teresis disappears. The intervals of allowed values for the light beam half-width $a$ and the NLC parameters $k$ and $m$, at which the LIFT is accompanied by the hysteresis become narrower with the increase of the electric field strength $E_{0}$.

\subsection{Electric field perpendicular to the cell surface}

Let the external dc electric field $E_{0}$ be oriented along the homogeneous NLC director, i.e. perpendicularly to the cell surface. The variational equation for the director deviation angle $\varphi$ looks like Eq. (4), in which the sign plus in front of the last term on the left-hand side is changed to the sign minus. The solution of the new equation for the director is sought in the form of the series expansions (6) and (7), similarly to the case

ISSN 2071-0194. Ukr. J. Phys. 2016. Vol. 61, No. 2 
where the dc electric field strength $E_{0}$ was oriented along the cell surface.

According to the results of numerical calculations, the dc electric field $E_{0}$ oriented perpendicularly to the cell surface does not affect the qualitative character of the dependences of the LIFT thresholds $I_{\mathrm{th}}$ and $I_{\mathrm{th}}^{\prime}$, and the LIFT hysteresis loop width $\Delta I_{\mathrm{th}}$ on the transverse light beam size, as was in the case $\mathbf{E}_{0} \| O x$. With the increase of the electric field strength $E_{0}$, the magnitudes of both LIFT thresholds $I_{\text {th }}$ and $I_{\text {th }}^{\prime}$, and the LIFT hysteresis loop width $\Delta I_{\text {th }}$ monotonically grow (Fig. 5), since the electric field stabilizes the initial director orientation. The hysteresis loop width as a function of the light beam width has a maximum, as was in the case $\mathbf{E}_{0} \| O x$. The maximum position shifts toward narrower light beams with the increase of the $E_{0}$-strength values.

For the sake of comparison, Fig. 5 also demonstrates the hysteresis loop widths $\Delta I_{\text {th }}$ experimentally measured for various NLCs in incident light beams with finite transverse sizes, which were taken from works [18, 20-22]. In order to broaden the LIFT hysteresis loop, the cited authors applied external dc electric [20-22] and magnetic [18] fields along the direction of the homogeneous NLC director. According to calculations, the electric field, which is equivalent to the magnetic one used in work [18], amounts to $E_{0}=1.35 E_{0 \mathrm{Fr}}$. In works $[18,20-22]$, the NLC anchoring at the cell surface was considered infinitely rigid. The measurement accuracy of the hysteresis loop width amounted to $\pm 10 \%$. In general, the results of theoretical calculations give a satisfactory agreement with experimental data. A certain difference between the experimentally measured and calculated values of the dc electric field strength $E_{0}$ is explained by the fact that, although the reorientation of the NLC director was considered in works $[18,20-$ 22 ] to be planar, actually, the light beams confined in two dimensions and with a non-uniform intensity distribution over their transverse cross-sections were used in the experiment.

The dependences of the critical light beam halfwidth $a_{\text {th }}$ on the electric field strength $E_{0}$ calculated for various values of parameter $k$ are shown in Fig. 3, b. Now, the interval of allowed values for the light beam half-width $a$, at which the LIFT is accompanied by the hysteresis $\left(a>a_{\mathrm{th}}\right)$, broadens with the growth of the electric field strength $E_{0}$, unlike the case $\mathbf{E}_{0} \| O x$. The increase of the parameter $k$

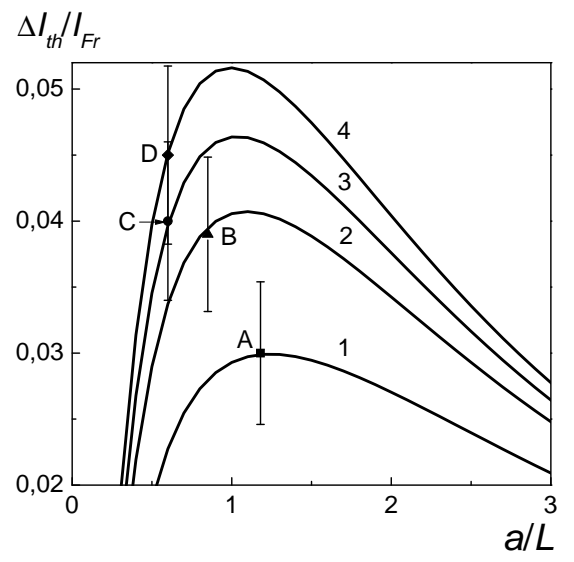

Fig. 5. Hysteresis loop width $\Delta I_{\text {th }}$ versus the half-width $a$ of a light beam with $I(y)=I_{0} \Theta(a-|y|)$ for $E_{0} / E_{0 \mathrm{Fr}}=$ $=0.975$ (1), 1.0 (2), 1.085 (3), and 1.115 (4). $k=0.38$ and $m=0.3$. Experimental points: $5 \mathrm{CB}-\mathrm{A}\left(E_{0} / E_{0 \mathrm{Fr}}=\right.$ $=1.35)$ [18]; ROTN-200 - B [22], C $\left(E_{0} / E_{0 F r}=1.57\right)$ [20], D $\left(E_{0} / E_{0 \mathrm{Fr}}=1.23\right)[21]$

results in the expansion of the interval for the values of transverse light beam size, at which the LIFT hysteresis occurs, irrespective of the orientation and magnitude of the dc electric field strength.

In Fig. $4, b$, the dependences of the critical values of parameter $m$ on the electric field strength $E_{0}$ calculated for various transverse sizes of the light beam are shown. The intervals of the allowed values for both parameters $m$ and $k$ of the liquid crystal, at which the LIFT is accompanied by the hysteresis, grow with the increase of the dc electric field strength $E_{0}$, unlike the case where this field was oriented along the cell surface. With the increase of the transverse light beam size, the $k$ - and $m$-intervals of the LIFT hysteresis existence expand irrespective of the orientation and magnitude of the dc electric field strength.

Hence, as the strength $E_{0}$ of the external dc electric field oriented normally to the cell surface increases, the LIFT threshold magnitudes $I_{\mathrm{th}}$ and $I_{\mathrm{th}}^{\prime}$, and the LIFT hysteresis loop width $\Delta I_{\text {th }}$ monotonically grow, and the intervals of the allowed values of light beam half-width $a$ and the NLC parameters $k$ and $m$ at which the LIFT is accompanied by the hysteresis get wider.

The authors express their gratitude to I.P. Pinkevych for useful remarks while discussing the results of this work. 
1. L.M. Blinov and V.G. Chigrinov, Electrooptic Effects in Liquid Crystal Materials (Springer, New York, 1994).

2. I.-C. Khoo, Liquid Crystals (Wiley, Hoboken, NJ, 2007).

3. Y.-Ki Ha,Y.-C. Yang, J.-E. Kim, H. Y. Park, C.-S. Kee, H. Lim, and J.-C. Lee, Appl. Phys. Lett. 79, 15 (2001).

4. M. Peccianti, C. Conti, G. Assanto, A. de Luca, and C. Umeton, Appl. Phys. Lett. 81, 3335 (2002).

5. M.J. Escuti, J. Qi, and G.P. Crawford, Opt. Lett. 28, 522 (2003).

6. R. Ozaki, T. Matsui, M. Ozaki, and K. Yoshino, Appl. Phys. Lett. 84, 1844 (2004).

7. B. Maune, M. Loncar, J. Witzens, M. Hochberg, T. BaehrJones, D. Psaltis, and A. Scherer, Appl. Phys. Lett. 85, 360 (2004).

8. D. McPhail, M. Straub, and M. Gu, Appl. Phys. Lett. 86, 051103 (2005).

9. A. Fratalocchi, G. Assanto, K.A. Brzdakiewicz, and M.A. Karpierz, Opt. Express 13, 1808 (2005).

10. S.P. Palto, Usp. Fiz. Nauk 175, 784 (2005).

11. A.S. Zolot'ko, V.F. Kitaeva, N. Kroo, N.N. Sobolev, and L. Chillag, Pis'ma Zh. Eksp. Teor. Fiz. 32, 170 (1980).

12. S.D. Durbin, S.M. Arakelian, and Y.R. Shen, Phys. Rev. Lett. 47, 1411 (1981).

13. B.Ya. Zeldovich, N.V. Tabiryan, and Yu.S. Chilingaryan, Zh. Èksp. Teor. Fiz. 81, 72 (1981).

14. I.C. Khoo, Phys. Rev. A 23, 2077 (1981).

15. B.Ya. Zeldovich and N.V. Tabiryan, Zh. Èksp. Teor. Fiz. 82, 1126 (1982).

16. H.L. Ong, Phys.Rev. A 28, 2393 (1983).

17. H.L. Ong, Phys. Rev. A 31, 3450 (1985)

18. A.J. Karn, S.M. Arakelian, Y.R. Shen, and H.L. Ong, Phys. Rev. Lett. 57, 448 (1986).

19. P.-Y. Wang, H.-J. Zhang, and J.-H. Dai, Opt. Lett. 12, 654 (1987).

20. S.-H. Chen and J.J. Wu, Appl. Phys. Lett. 52, 1998 (1988).

21. J.J. Wu and S.-H. Chen, J. Appl.Phys. 66, 1065 (1989).

22. J.J. Wu, G.-S. Ong, and S.-H. Chen, Appl. Phys. Lett. 53, 1999 (1988).

23. A.S. Zolot'ko, M.P. Smayev, V.F. Kitaeva, and M.I. Barnik, Quant. Electr. 34, 1151 (2004).

24. I. Budagovsky, D. Pavlov, S. Shvetsov, M. Smayev, A. Zolot'ko, N. Boiko, and M. Barnik, Appl. Phys. Lett. 101, 021112 (2012).

25. B.J. Frisken and P. Palffy-Muhoray, Phys. Rev. A 40, 6099 (1989).

26. H.L. Ong, R.B. Meyer, and A.J. Hurd, J. Appl. Phys. 55, 2809 (1984)
27. G. Abbate, P. Maddalena, L. Marrucci, L. Saetta, and E. Santamato, J. Phys. II (Paris) 1, 543 (1991).

28. B. Piccirillo and E. Santamato, Phys. Rev. E 69, 056613 (2004).

29. V. Ilyina, S.J. Cox, and T.J. Sluckin, Opt. Commun. 260, 474 (2006).

30. E.A. Babayan, I.A. Budagovsky, S.A. Shvetsov, M.P. Smayev, A.S. Zolot'ko, N.I. Boiko, and M.I. Barnik, Phys. Rev. E 82, 061705 (2010).

31. A.A. Berezovskaya, S.N. Yezhov, M.F. Ledney, and I.P. Pinkevych, Funct. Mater. 14, 510 (2007).

32. M.F. Ledney and A.S. Tarnavskyy, Kristallogr. 55, 337 (2010).

33. M.F. Ledney and O.S. Tarnavskyy, Ukr. Fiz. Zh. 56, 30 (2011).

34. J. Shi and H. Yue, Phys. Rev. E 62, 689 (2000).

35. M.F. Ledney and O.S. Tarnavskyy, Liq. Cryst. 39, 1482 (2012).

36. B.J. Frisken and P. Palffy-Muhoray, Phys. Rev. A 39, 1513 (1989).

Received 07.07.15.

Translated from Ukrainian by O.I. Voitenko

М.Ф. Ледней, О.С. Тарнавсъкий, В.В. Хіміч

ВПЛИВ СТАТИЧНОГО ЕЛЕКТРИЧНОГО

ПОЛЯ НА ГІСТЕРЕЗИС СВІТЛОІНДУКОВАНОГО ПЕРЕХОДУ ФРЕДЕРІКСА В НЕМАТИЧНІЙ КОМІРЦІ

$\mathrm{P}$ е $з$ ю м е

Розглянуто вплив величини напруженості зовнішнього статичного електричного поля на гістерезис світлоіндукованого переходу Фредерікса в комірці нематичного рідкого кристала в полі світлового пучка з обмеженим поперечним розміром. Розглянуті випадки орієнтації зовнішнього електричного поля перпендикулярно поверхні комірки та вздовж цієї поверхні в напрямку вектора поляризації падаючого світла. Чисельно отримані значення порогів переходу Фредерікса при збільшенні і зменшенні інтенсивності падаючого світла в залежності від величини напруженості електричного поля і поперечного розміру світлового пучка. Визначені області допустимих ширин світлового пучка і значень напруженості електричного поля, під час яких перехід Фредерікса супроводжується гістерезисом. Показано, що ширина петлі гістерезису зростає зі збільшенням напруженості статичного електричного поля, перпендикулярного до поверхні комірки, і зменшується, якщо електричне поле напрямлене вздовж поверхні комірки. 